\section{e0214 N-ACETYLCYSTEINE INHIBITS OXIDISED LDL, METALLOPROTEINASES -9 SERCRETION AND APOPTOSIS IN ATHEROSCLEROSIS}

doi:10.1136/hrt.2010.208967.214

Li Zhenxiao, Meng Xiaoping. Cardiology, The Second Hospital of Jilin University, China

Atherosclerosis is a complex disease, in which oxidative stress in atherosclerosis through the whole process, antioxidant treatment is imperative.

Objective Discuss the effect of $\mathrm{N}$-acetyl cysteine (NAC) in the APOE gene knockout mice in anti-atherosclerosis.

Method In this study, select 8-week-old male Apoe knockout (Apoe-/-) mice were studied, a total of 10, with an average weight of $20 \mathrm{~g}$. Were randomly divided into high-fat diet group (control group), and NAC interfere with treatment group (experimental group), two groups of mice were treated with high-fat diet, NAC intervention group administered a dose of $300 \mathrm{mg} / \mathrm{kg}$, according to body weight of mice in each of the group on the required dose, ministered through drinking water bottle means, and to ensure that set up a daily dose of drug intake. Modelling 60 days, to give a 24-h fasting mice, pick the eye blood, placed in test tubes containing EDTA-specific biochemical tests carried out centrifugation, separated plasma. Off mice were sacrificed, rapid separation of mouse aortic arch and access to paraffin-embedded specimens. By using situ immunofluorescence staining method for the determination of artery intima-mouse levels, to observe the protection of NAC in atherosclerosis during the course of apoptosis; in serological testing, the choice of ELISA method was determined to assess the plasma MMP-9 in mice with ox-LDL levels; artery atherosclerotic plaques in the general morphological study using haematoxylin - eosin (HE) staining method, histological specimens of atherosclerotic lesions in general shape.

Result In the general model of atherosclerosis, the visual plaque morphology: NAC experimental group of mice the distribution of atherosclerotic plaque area less than high-fat diet group; HE stained specimens, NAC group foam cells were markedly reduced plaque in the volume of foam cells and plaque lipid core size significantly decreased compared with the control group; by in situ fluorescence staining method, determination of arterial intima in the expression of MMP-9 positive cells, NAC group of positive cells $(6.00 \pm 2.45)$ than the control group $(20.08 \pm 5.17)(p<0.05)$, statistically significant; measured the number of apoptotic cells, NAC group number of apoptotic cells $(7.80 \pm 1.07 \%)$ than the control group $(9.22 \pm 0.84 \%)(p<0.05)$, statistically significant; NAC group plasma ox-LDL levels $(9.89 \pm 1.33 \mu \mathrm{g} /$ dl) than the control group $(15.84 \pm 2.60 \mu \mathrm{g} / \mathrm{dl})(\mathrm{p}<0.05)$, NAC group plasma MMP-9 levels $(8.50 \pm 1.58 \mathrm{ng} / \mathrm{ml})$ than the control group $(12.44 \pm 2.19 \mathrm{ng} / \mathrm{ml})(\mathrm{p}<0.05)$, were statistically significant.

Conclusion NAC can reduce the number of atherosclerotic plaque, plaque distribution area, reducing the number of foam cells, reducing the volume of foam cells in plaques, plaque lipid core volume; NAC can reduce the arterial endometrial expression of MMP-9 in the number of positive cells; NAC can reduce atherosclerotic plaques in the number of apoptotic cells; NAC can reduce plasma ox-LDL and MMP-9 levels. This experiment further confirmed the NAC's antiatherosclerosis effect of NAC as an anti-atherosclerosis drugs used clinically to provide an experimental basis.

\section{e0215 ENHANCED EXTERNAL COUNTERPULSATION PROTECTS VASCULAR ENDOTHELIAL CELLS FROM APOPTOSIS IN HYPERCHOLESTEROLEMIC PIGS}

doi:10.1136/hrt.2010.208967.215

Yan Xiong. Division of Emergency Medicine, First Affiliated Hospital, Sun Yat-sen University, Guangzhou, China

Objective Evidences have proved that Enhanced external counterpulsation (EECP) improves endothelial dysfunction and repairs intimal damage by increasing vascular endothelial shear stress. Based on the assumption that unbalanced apoptosis of vascular endothelial cells (VECs) may have played a pivotal role in the pathogenesis of atherosclerotic lesions, we hypothesised that long-term EECP protects VECs from apoptosis in hypercholesteronlemic pigs.

Methods 18 male domestic pigs were randomly assigned to three groups: one normal control group with a normal diet (Normal, $n=6$ ) and two hypercholesterolemic groups $(\mathrm{HC}, \mathrm{n}=12)$ fed with atherosclerosis-inducing cholesterol-rich chow diet, one of which received enhanced external counterpulsation (HC, $\mathrm{HC}+\mathrm{EECP}, \mathrm{n}=6$, respectively). Pigs in the $\mathrm{HC}+\mathrm{EECP}$ group were treated with EECP for $2 \mathrm{~h}$ every other day for a total of $36 \mathrm{~h}$. In the end of the study, the animals were sacrificed, and the thoracic and abdominal aortas harvested. The thoracic aortas were sampled for both scanning and transmission electronic microscopy whereas the abdominal aortas were stained in Sudan-III of fatty streak for macroscopic evaluation. Meanwhile, vascular endothelial cells (VECs) were isolated from the thoracic aorta by the use of collagenase. TUNEL method was used to detect the apoptotic index of VECs. At the same time, the abdominal aortas were collected for histopathological studies.

Results Fatty streaks or plaques, positively stained by Sudan dye, were hardly found in the normal group but clearly observable in the $\mathrm{HC}$ group. And among the cholesterol-diet animals, atherosclerotic lesions were much less severe in the EECP group than in the HC group. Scanning electronic microscopic analysis revealed that aortic VECs were irregularly arrayed, markedly desquamated, and shrank into smaller size, which indicated apoptotic events resulting in remarkable damage of endothelium in $\mathrm{HC}$ group. In contrast, the VECs in HC+EECP group were arrayed in a relatively streamline fashion, less desquamated and shrank, and manifested comparatively mild endothelial damage. Transmission electronic microscopic examination of aortas in HC group showed desquamated VECs loosely attached to the matrix along with foam cells, which indicated intimal damage. Apoptotic VECs at early, middle, late stage and even apoptotic bodies were visible on intimal surface. Nonetheless, these changes were relatively mild in EECP-treated animals. Meanwhile, the apoptotic index in the HC+EECP group was significantly lower than that of the HC group, but still higher than that of the Normal group $((177 \pm 12) \%$, $(237 \pm 23) \%$, $(127 \pm 36) \%$, respectively, $\mathrm{p}<0.05$ ).

Conclusions EECP alleviates hypercholesterolaemia-induced atherosclerotic damage to the vascular intima and endothelium, and protects vascular endothelial cells from apoptosis, thereby delaying the progression of early atherosclerotic lesions. The therapeutic benefit of EECP in terms of endothelial protection may be attributed to the inhibition of VEC apoptosis.

\section{e0216 THE EXPRESSION AND ROLE OF INTERLEUKIN-23 IN MICE VIRUS MYOCARDITIS}

doi:10.1136/hrt.2010.208967.216

Fan Yang, Weifeng Wu, Yuluan Yan, Qing Kong, Yu Pang. The First Affiliated Hospital of Guangxi Medical University, Nanning, China

Objective To explore the expression of IL-23 and its role in acute viral myocarditis mice induced by coxsackie virus B3 (CVB3).

Methods To establish the model of $\mathrm{VMC}, \mathrm{Balb} / \mathrm{c}$ male mice were peritoneally injected (IP) with Coxsackievirus B3, mice peritoneally injected with PBS were taken as the controls. On 0, 1, 2, 3, 4 and 6W after IP, haematoxylin-eosin was used to assess pathological changes. The level of IL-23 mRNA in mice myocardic tissue was determined by RT-PCR. The expression of IL-23 protein in blood plasma was evaluated by EILSA.

Results Comparing with the controls, the expression of IL-23 mRNA were steady high from $1 \mathrm{~W}$ after IP, and maintaining a higher trend until 6W. All the results at different time were higher than 